\title{
Transmenschen brauchen ihren Platz im Gesundheitswesen
}

Isabel Zwyssig

M.A., freie Journalistin
In diesem Text steht der Gendergap, eine durch einen Unterstrich gekennzeich nete Lücke zwischen der maskulinen Form und der femininen Endung eines Wortes. Der Gendergap widerspiegelt die sprachliche Gleichbehand lung und Repräsentation aller sozialen Geschlechter

Niklaus Flütsch, Facharzt mit Spezialgebiet gynäkologische Endokrinologie in Zug, betreut erwachsene und jugendliche Transpersonen. Diesen Menschen, deren erlebte Geschlechtsidentität nicht mit ihren körperlichen Geschlechtsmerkmalen übereinstimmt, helfen verschiedene chirurgische und hormonelle Behandlungsmethoden. Obwohl die WHO Transmenschen in der neuen ICD-11 nicht mehr als psychisch krank klassifiziert und die Gesellschaft ihnen seit den letzten Jahrzehnten toleranter begegnet, muss das Verständnis für Transpersonen weiter gefördert werden - auch im Gesundheitswesen.

Es gibt Menschen, die das biologische Geschlecht, das ihnen bei der Geburt zugeordnet worden war, nicht mit dem seelischen Erleben ihrer geschlechtlichen Identität in Einklang bringen: Transpersonen. Die Diskrepanz zwischen angeborenen anatomischen Merkmalen und geschlechtlichem Empfinden kann je nach Lebensbereich ganz unterschiedliche Ausmasse annehmen. Geraten Transmenschen über einen längeren Zeitraum unter starken psychischen und sozialen Druck, unter dem sie zunehmend leiden, so erhält ihr Zustand Krankheitswert. Man spricht in diesem Fall von einer Geschlechtsdysphorie [1].

\section{Transpersonen in der Schweiz: Es gibt eine Dunkelziffer}

Für die Schweiz sind keine genauen Zahlen bekannt, die Aussagen über die Häufigkeit von Transidentität machen. Statistisch erfasst werden nur jene Transmenschen, die fachliche Hilfe in Anspruch nehmen. «Nach gegenwärtigem wissenschaftlichen Kenntnisstand ist es sehr wahrscheinlich, dass schon immer Menschen existiert haben, die ihre physischen Geschlechtscharakteristika als nicht übereinstimmend mit ihrem Erleben erfahren", erklärt Niklaus Flütsch, Facharzt für gynäkologische Endokrinologie und Leiter der Spezialsprechstunde für transidente Menschen am Zürcher Stadtspital Triemli. Erst seit Anfang des 20. Jahrhunderts beschäftigt sich die Medizin mit dem Phänomen der Geschlechtsinkongruenz - so der neutrale Begriff im Gegensatz zur Geschlechtsdysphorie, die aufgrund des Leidensdrucks eine pathologische Dimension impliziert [2].

\section{Transmenschen suchen heute früher Hilfe}

Oft spüren Transmenschen seit früher Kindheit, dass zwischen ihren körperlichen Geschlechtseigenschaften und ihrem erlebten Männlich- oder Weiblichsein eine grosse Kluft existiert. Laut dem Basler Psychotherapeuten Patrick Gross erlangt ein Kind zwischen fünf und sechs Jahren ein dauerhaftes Bewusstsein dafür, ob es ein Mädchen oder ein Junge ist [3]. Wie lange und schmerzvoll der Weg von Transmännern und -frauen sein kann - vor allem falls von einer Geschlechtsdysphorie betroffen -, lässt sich in Anbetracht dieser Tatsache lediglich erahnen. Auf welchen Wegen Transpersonen ins Gesundheitssystem gelangen, ist unterschiedlich. Viele von ihnen öffnen sich zunächst ihren Grundversorger_innen, weshalb es sinnvoll ist, dass diese behutsam an das Thema Transidentität herangehen [1]. Wenn Transfrauen und Transmänner Niklaus Flütsch begegnen, sind ihre Bedürfnisse sehr individuell. «Von Personen mit klaren Vorstellungen bis Suchenden ist alles dabei.» Patient_innen konsultieren Niklaus Flütsch bisweilen für eine Zweitmeinung. «Die meisten Transmänner und -frauen schätzen Wirkungen von geschlechtsangleichenden Interventionen wie Hormontherapie und Operationen erstaunlich realistisch ein. Ebenso verhält es sich mit Erwartungen an das körperliche Funktionieren sowie das Leben mit 


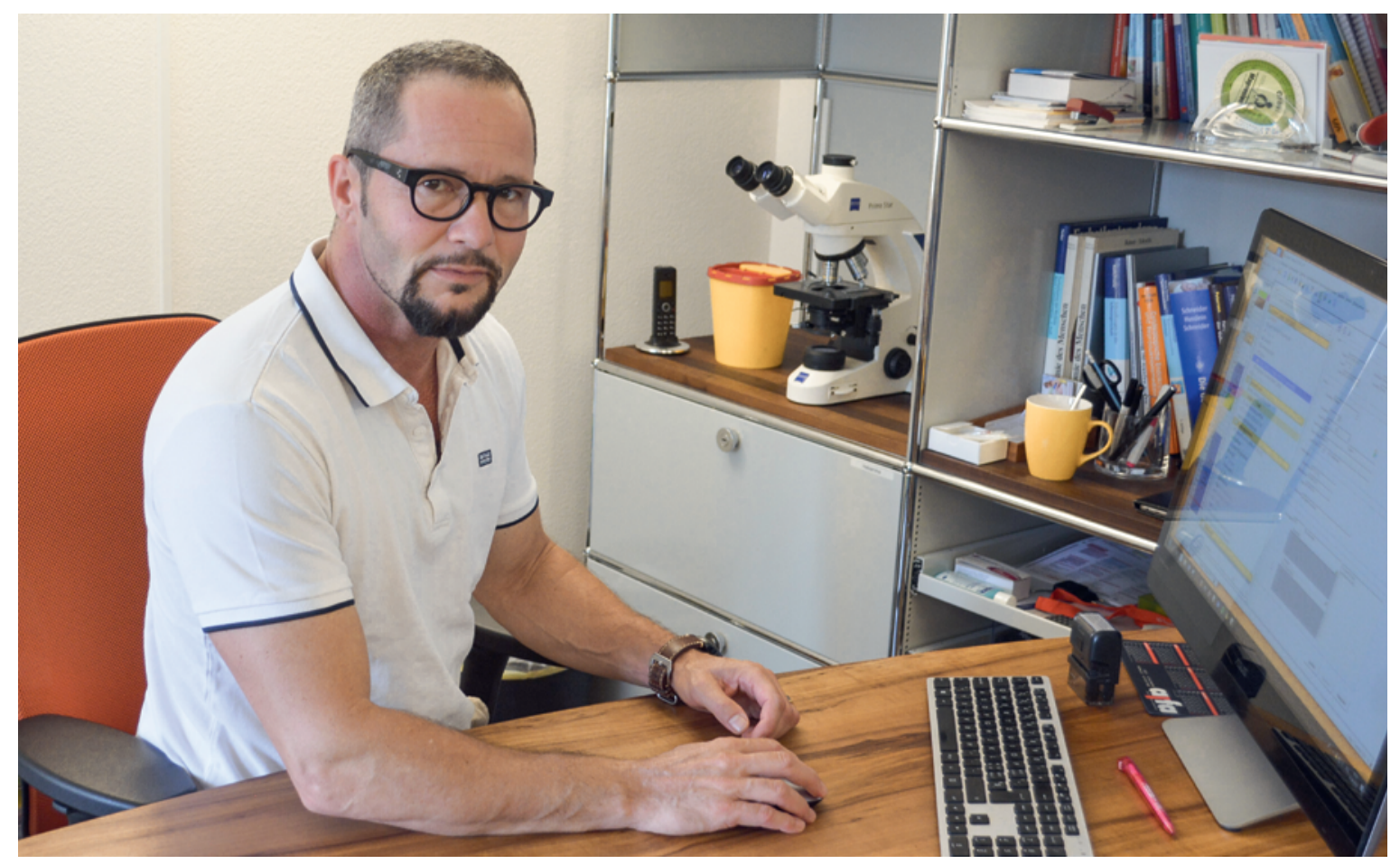

Niklaus Flütsch bietet eine Transgender-Sprechstunde an - auch Kinder und Jugendliche sind herzlich willkommen.

angepassten äusseren Geschlechtsmerkmalen", so der Facharzt. Entscheidend ist bei jeder Beratung, dass die Patient_innen psychisch stabil, urteilsfähig und in der Lage sind, die Konsequenzen der Behandlungen abzuschätzen sowie auf der Grundlage ihres Ermessens eine sinnvolle Entscheidung zu treffen. Klinische Erfahrungen belegen, dass geschlechtsangleichende Behandlungen die genderdysphorische Symptomatik verringern und die Lebensqualität deutlich optimieren [1]. Auch Kinder und Jugendliche im Alter von 9 bis 14 Jahren kommen in Niklaus Flütschs Transgender-Sprechstunde. Anders als bei Erwachsenen muss bei ihnen das soziale Umfeld stärker mitbetreut werden. Denn: Wie persistent der Wunsch ist, im anderen Geschlecht zu leben, zeigt sich gerade bei Kindern erst später. Tendenziell sind die Patient_innen jünger geworden. Niklaus Flütsch: «Es ist erfreulich, dass viele heute nicht mehr bis zu ihrem 50. Lebensjahr warten, bis sie Hilfe suchen.»

\section{Frage nach Kinderwunsch sorgfältig klären}

Gemäss den Behandlungsempfehlungen der World Professional Association for Transgender Health (WPATH) setzt die Hormonabgabe an Transmenschen seit 2011 keine psychotherapeutische Begleitung mehr voraus. In der Regel beginnen Patient_innen zuerst mit der einschneidenden Hormontherapie, bevor sie operative geschlechtsangleichende Massnahmen in Betracht ziehen. Abweichungen gibt es jedoch durchaus. «Nicht bei allen Menschen schlägt die Hormonbehandlung gleich schnell an», erklärt Niklaus Flütsch. «Diese Transfrauen und -männer haben tendenziell mehr Mühe, die nötige Geduld aufzubringen, bis ihr

«Es ist erfreulich, dass viele heute nicht mehr bis zu ihrem 50. Lebensjahr warten, bis sie Hilfe suchen.»

Körper reagiert. Bei einem Transmann mit üppiger Brust beispielsweise kann es unter Umständen vertretbar sein, zuerst eine Mastektomie durchzuführen, bevor er mit der Hormonbehandlung anfängt.» Wollen Patient_innen zu schnell zu viel, mahnt Niklaus Flütsch zur Vorsicht. Insbesondere dann, wenn sie im reproduktionsfähigen Alter sind und die Frage nach einem Kinderwunsch zu klären ist.

\section{Operative Behandlungsmöglichkeiten für Transmänner}

Chirurgische Massnahmen, die für Transmänner zur Verfügung stehen, beinhalten einen vielschichtigen, anspruchsvollen Prozess, der etappenweise durchgeführt wird. Der erste Schritt ist meist die Entfernung 
der weiblichen Brust. Auf diese Weise kann der Patient sein Körperbild leichter in seine männliche Geschlechtsidentität integrieren. Danach folgen in der Regel Hysterektomie und Ovarektomie. Zu diesem Zeitpunkt der Transition ist die Klitoris durch die Testosteroneinwirkung gewachsen; sie ist sichtbarer und beweglicher. Indem sie operativ freipräpariert wird, kann nun die Harnröhre durch den Klitorispenoid gelegt werden. Die Scheide wird verschlossen. Dieser kleine Penisaufbau bedeutet entweder einen Zwischenschritt oder die vorläufig letzte Hürde für jene Transmänner, die - nicht selten aus Furcht, ihre erogene Empfindungsfähigkeit zu verlieren - künftig darauf verzichten, zu penetrieren. Allerdings: «Einen Penoid wünschen sich viele Transmänner nicht primär für Sex. Es geht ihnen darum, ein Körpergefühl zu haben, das mit ihrer männlichen Geschlechtsidentität kongruent ist. Sie wollen im Stehen urinieren können", betont Niklaus Flütsch. Für Transmänner, die ihre Geschlechtsangleichung weiter vollziehen möchten, besteht die Option eines grossen Penis-

\section{Da es sich bei der Transgender-Medizin um} eine hochspezialisierte Randdisziplin handelt, ist sie weltweit vernetzt.

aufbaus. Dazu dienen Haut und Gewebe von anderen Stellen des Körpers - meistens vom Unterarm für den Aufbau des Penoids, dessen Basis die hypertrophierte Klitoris ist. Die Chirurgen schliessen den Penoid an die vorhandenen Nerven an, mit dem Ziel, die Sensibilität wiederherzustellen und die Orgasmusfähigkeit zu erhalten. Dank einer Pumpe, die auf Knopfdruck den Penis versteift, können diese Transmänner penetrieren $[4,5]$.

\section{Chirurgische Therapieoptionen für Transfrauen}

Auch bei Transfrauen stellt aus Gründen des stimmigeren Körperbildes die Brustchirurgie oft der erste Schritt dar. Hat die Brust nicht bereits dank der Hormontherapie das gewünschte Volumen erlangt, kann sie durch Implantate vergrössert werden. Aus der Haut von Hoden und Penis, die zuvor entfernt worden sind, werden Vagina, Klitoris, äussere sowie zum Teil innere Schamlippen gefertigt. Empfindungs- und Orgasmusfähigkeit sind bei dieser Methode sichergestellt. Logopädie oder eine Stimmbandverkürzung helfen Transfrauen, an einer helleren stimme $\mathrm{zu}$ arbeiten. Chirurgisch können ausserdem femininere Gesichtszüge geschaffen werden [5].

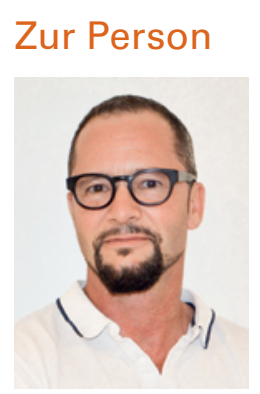

Niklaus Flütsch ist selbständiger Facharzt für Gynäkologie und Geburtshilfe mit einer Praxis in Zug. Am Zürcher Stadtspital Triemli leitet er eine Spezialsprechstunde für transidente Menschen. 2014 publizierte Niklaus Flütsch seine Autobiografie Geboren als Frau Glücklich als Mann. Logbuch einer Metamorphose, in der er in Zusammenarbeit mit der Autorin Ursula Eichenberger seine persönlichen Erfahrungen mit dem Transsein aufzeichnet. Der Gynäkologe verfasst Fachartikel, hält Vorträge und bietet Workshops an, um die Öffentlichkeit für das Thema Transidentität zu sensibilisieren.

Da es sich bei der Transgender-Medizin um eine hochspezialisierte Randdisziplin handelt, wo verschiedene medizinische Fachbereiche wie Gynäkologie, Urologie, Endokrinologie und Psychiatrie ineinandergreifen, ist sie weltweit vernetzt. Anfang September 2018 ist Basel Schauplatz eines wichtigen Meetings geworden: Hier haben sich erstmals internationale Experten zusammengefunden, um über eine Standardisierung der geschlechtsangleichenden Chirurgie zu beraten. Im Anschluss an das Treffen werten die Fachleute ihre Ergebnisse aus. Zum Zeitpunkt der Niederschrift dieses Artikels existieren dazu noch keine Richtlinien

\section{Transmenschen gelten nicht mehr als psychisch krank}

«Wie weit die einzelnen Transfrauen und Transmänner in ihrer Geschlechtsangleichung gehen, ist abhängig von persönlichen Vorstellungen unterschiedlich", betont Niklaus Flütsch. "Manche Transmenschen leben ihre angeborene Geschlechtsidentität im biologisch gegengeschlechtlichen Körper aus. Sie arrangieren sich mit der Situation - ohne Operationen.» Seit der deutsch-amerikanische Sexualwissenschaftler Harry Benjamin durch seine 1966 veröffentlichte Monografie The transsexual phenomenon das Thema erstmals in den Fokus der wissenschaftlichen Aufmerksamkeit der damaligen Medizin rückte [2], hat sich die Situation von Transmenschen in vielen Bereichen des gesellschaftlichen Lebens verbessert. Einer der grössten Erfolge dürfte sicherlich die längst fällige Entpsychopathologisierung darstellen. Galten Transpersonen in den 80er Jahren noch als persönlichkeitsgestört, so bezeichnet die WHO in der überarbeiteten Fassung ihres internationalen Klassifikationssystems für Krankheiten Transmenschen nicht mehr als 
psychisch beeinträchtigt oder verhaltensauffällig. Der veraltete diagnostische Begriff «Transsexualität» findet keine Verwendung mehr. Neu spricht man von Gender Incongruence (Geschlechtsinkongruenz). Schwierigkeiten, mit denen Transmenschen konfrontiert sind, werden im Bereich der sexuellen Gesundheit verortet. Am 1. Januar 2022 soll die neue ICD-11 in Kraft treten [6]. Auch in den Therapieleitlinien der

\section{Obwohl die Gesellschaft der Geschlechterviel- falt gegenüber immer toleranter geworden ist, müssen weiterhin Tabus aufgebrochen werden.}

WPATH gibt es beachtliche Veränderungen zu verzeichnen. Eine davon ist etwa die Abschaffung des "Alltagstests». Bis 2012 waren Transmenschen dazu verpflichtet, während mindestens eines Jahres in die Rolle des gewünschten Geschlechts zu schlüpfen, bevor sie medizinische Massnahmen zur Geschlechtsangleichung einleiten durften. In dieser "Generalprobe» vor der Geschlechtsanpassung sollten sie ihre Geschlechtsidentität auf dem gesellschaftlichen Parkett erproben.

\section{Es braucht eine einheitliche Krankheits- definition im KVG}

Heute müssen sich Transfrauen und Transmänner Mediziner_innen gegenüber nicht mehr «beweisen», um bestimmte Behandlungen zu erhalten. Bei der gesundheitlichen Versorgung stehen ihre persönlichen Bedürfnisse im Vordergrund. Handlungsbedarf sieht Niklaus Flütsch jedoch beim Krankenversicherungsgesetz und beim damit verbundenen Umgang der Krankenkassen mit Versicherungsleistungen für Transmenschen. Problematisch ist, dass die Krankheitsdefinition der Geschlechtsdysphorie nicht einheitlich gehandhabt wird. Kosten für Anpassungen primärer und sekundärer Geschlechtsmerkmale, die in der Schweiz durchgeführt werden, sind in der Regel durch die Grundversicherungen gedeckt. Weil jedoch im KVG keine konforme Definition der Geschlechtsdysphorie verankert ist, kann es vorkommen, dass gewisse Kosten je nach Krankenkasse nicht übernommen werden. Obwohl sich die Gesellschaft im Laufe der Jahre Geschlechtervielfalt gegenüber immer toleranter gezeigt hat, müssen weiterhin Tabus aufgebrochen werden - auch im schweizerischen Gesundheitswesen. Gerade dort sind Transpersonen darauf angewiesen, dass Fachleute ihre Anliegen ernst nehmen.

\section{Unser Gesundheitssystem ist nicht auf Transpersonen vorbereitet}

David Garcia, leitender Arzt des Zentrums für Geschlechtervarianz am Universitätsspital Basel, beobachtet, dass Transmenschen in der Schweiz medizinisch unterversorgt sind [1]. Medizinisches Fachpersonal und Ärzt_innen reagieren auf die hohen Anforderungen, die Beratung, Behandlung und Nachbetreuung von Transpersonen an sie stellen, verständlicherweise nicht immer adäquat, weil Erfahrung und Wissen fehlen. Obgleich für viele Lebensbereiche nicht relevant, spielen biologisches und soziales Geschlecht in manchen Kontexten durchaus eine Rolle. "Hier ist es sinnvoll, das Konstrukt Geschlecht zu differenzieren", ist David Garcia überzeugt. "Wird die Kategorie trans bei einer medizinisch posttransitionierten Transfrau nicht erfasst, läuft sie Gefahr, nie urologisch untersucht zu werden. Dasselbe gilt für Transmänner, welche keine Hysterektomie haben durchführen lassen. Sie brauchen gynäkologische Betreuung. Diese erhalten sie nur, wenn sie als Transmänner wahrgenommen werden.» Niklaus Flütsch ist der Meinung, dass wir unsere gegenwärtige, allgemeingültige Konzeption von Geschlecht überdenken, vor allem aber nicht zu eng sehen sollten. «Geschlecht stelle ich mir wie eine Wolke vor. Es ist etwas Unscharfes, das sich nicht in ein festgefügtes Raster zwängen lässt. Transmenschen blieben einige Unannehmlichkeiten erspart, wenn die Geschlechtszugehörigkeit von staatlicher Seite zur Privatsache gemacht würde.»

\section{Bildnachweis}

Fotos Isabel Zwyssig

\section{Literatur}

1 Garcia D, et al. Von der Transsexualität zur Gender-Dysphorie. Beratungs- und Behandlungsempfehlungen bei TransPersonen In: Schweiz Med Forum. 2014;14(19):382-7.

2 Flütsch N. Endokrinologische Behandlung der Geschlechtsdysphorie bei Menschen mit Geschlechtsinkongruenz. In: Journal für Klinische Endokrinologie und Stoffwechsel. 2015;8(2):42-8.

3 Blumer F. «Herr Gross, wann weiss ein Kind, ob es ein Bub ist oder ein Mädchen?» In: Fritz + Fränzi. Das Schweizer ElternMagazin. 28. März 2018. https://www.fritzundfraenzi.ch/erziehung/elternbildung/herr-gross-wann-weiss-ein-kind-ob-es-ein-bub-ist-oderein-madchen [Stand: 14. September 2018].

4 Bauquis O, et al. Geschlechtsangleichende Chirurgie. Von der Unkenntnis zum Vorurteil. In: Schweiz Med Forum. 2014;14(49):919-23.

5 Flütsch N. Geboren als Frau - Glücklich als Mann. Logbuch einer Metamorphose. Gockhausen: Wörterseh Verlag; 2014.

6 Transgender Network Switzerland: https://mailchi.mp/ a62686cf8973/medienmitteilung-who-icd-11 [Stand: 15. September 2018] 